\title{
Uso problemático de internet, cyberbullying y ciber-violencia de pareja en jóvenes universitarios*
}

\author{
Problematic internet use, cyberbullying and cyber dating \\ violence among university students
}

\author{
Fabiola Peña Cárdenas** \\ ORCID: 0000-0001-5895-0816 \\ Universidad Autónoma de Tamaulipas, \\ México \\ José Luis Rojas-Solís ${ }^{* * *}$ \\ ORCID: 0000-0001-6339-4607 \\ Benemérita Universidad Autónoma \\ de Puebla \\ Paola Valeria García-Sánchez
Benemérita Universidad Autónoma
de Puebla
}

Recibido: 17 de julio de 2017 Revisado: 6 de septiembre de 2017 Aceptado: 4 de diciembre de 2017

\section{Resumen}

Las Tecnologías de la Información y Comunicación se han desarrollado con rapidez integrándose en la vida cotidiana de los adolescentes y los jóvenes. Por ello, esta investigación con diseño no-experimental y transversal analizó las diferencias por sexo en el uso problemático del internet, el cyberbullying y la ciber-violencia de pareja; así como la posible relación entre las variables mencionadas. La muestra fue conformada por 456 estudiantes, procedentes de dos universidades mexicanas, con edades entre 17 y 30 años $(M=19,86$; DT = 2,12). Los resultados mostraron pocas diferencias entre hombres y mujeres; asimismo se presentaron correlaciones bajas positivas entre la mayoría de los indicadores de uso problemático de internet con ciberbullying; el uso problemático de internet y ciber-violencia de pareja; y entre ciberbullying y ciber-violencia de pareja. Se concluyó

Artículo de investigación. DOI: https://doi.org/10.15332/s1794-9998.2018.0002.01

Doctora en Aprendizaje y Cognición por la Universidad de Sevilla (España). Profesora-Investigadora en la Universidad Autónoma de Tamaulipas (México). Correo electrónico: fcardenas@docentes.uat.edu.mx

*.* Doctor en Psicología por la Universidad de Salamanca (España) y Profesor-Investigador en la Benemérita Universidad Autónoma de Puebla (México). Correo electrónico: joseluis.rojas@correo.buap.mx Correspondencia: José Luis Rojas-Solís, Facultad de Psicología, Benemérita Universidad Autónoma de Puebla. Correo electrónico: joseluis.rojas@correo.buap.mx. Dirección postal: Calle 4 Sur \#403, Centro Histórico, 72000. Puebla, (México).

.... Alumna de la licenciatura en Psicología por la Benemérita Universidad Autónoma de Puebla (México) y becaria del Programa "Beca para Prácticas. Programa de Verano de la Investigación Científica SEP-AMC 2017”, Folio: 4965565. Correo electrónico: paola.v.garcia.sanchez@gmail.com 
que las conductas violentas de jóvenes mediante nuevas tecnologías parecen generalizarse a las relaciones entre pares y de pareja. Se discuten los resultados destacando la necesidad de más estudios sobre estos fenómenos cada vez más presentes en jóvenes.

Palabras clave: Uso problemático del internet, cyberbullying, ciber-violencia de pareja, universitarios.

\section{Abstract}

The Information and Communication Technologies have developed at a fast pace, integrating themselves in the daily life of adolescents and young people. For this reason, this non-experimental cross-sectional study analyzed the gender differences in problematic Internet use, cyberbullying and cyber-violence, as well as the possible relationship between these variables. The sample consisted of 456 students, from two Mexican universities, aged between 17 and 30 years $(M=19,86$; DT $=2,12$ ). The results showed few significant differences between men and women; likewise, significant positive correlations were found between most of indicators of problematic Internet use with cyberbullying; between problematic Internet use and cyber dating violence; and between cyberbullying and cyber dating violence. It was concluded that violent behavior of young people through new technologies seems to be generalized to relationships with peers and couples. It was discussed the results highlighting the need for more studies on these phenomena increasingly present in young people.

Keywords: Problematic internet use, cyberbullying, cyber dating, college students.

\section{Antecedentes}

A partir de la instalación de la sociedad virtual, el fenómeno de la globalización y el comercio cibernético, las Tecnologías de la Información y Comunicación (TICS) se han desarrollado con rapidez debido a los numerosos avances científicos y crecientes demandas sociales, incluso desde el ámbito académico donde se han solicitado con frecuencia para la mejora del currículo (HerreraBatista, 2009).

Así, no es sorprendente que las poblaciones de adolescentes y jóvenes actualmente cuenten con una gran habilidad de apropiación y adaptación de estos medios (principalmente aquellas TICs con acceso a internet) para sus actividades académicas y sociales (Castaño-Muñoz \& Senges, 2011; Espinar \& López, 2009), donde uno de los principales usos del internet es el acceso a las redes sociales (Vázquez, 2016); tanto para menores de 17 años (Rial, Gómez, Braña \& Varela, 2014) como para jóvenes universitarios (Molero et al., 2014; Puerta-Cortés \& Carbonell, 2013).
Esta situación ha originado diversos cambios en los estilos de vida, como nuevas formas de relaciones de pareja, y comportamientos sociales (Carruth \& Ginsburg, 2014) en el que este medio es usado para obtener algún tipo de recompensa o, incluso, para aminorar las situaciones presenciales displacenteras (García et al., 2008). Sin embargo, pese a los beneficios que ofrecen, en determinadas situaciones, este recurso puede convertirse en un objetivo prioritario de obtención de placer que, asociado a variables como la vulnerabilidad psicológica y factores estresantes, pueden traer consigo el uso descontrolado de este medio (Odaci \& Kalkan, 2010; Puerta-Cortés \& Carbonell, 2014).

Por consiguiente, surge una controversia respecto a la tipificación de dichas conductas donde algunos investigadores sugieren que es posible caer en conductas adictivas (Arnaiz, Cerezo, Giménez \& Maquilón, 2016), mientras que otros prefieren identificarlas como conductas problemáticas (Fernández-Villa et al., 2015; Ruíz-Olivares, Lucena, Pino \& Herruzo, 2010); lo cierto es que ambas pos- 
turas coinciden en que es una necesidad evaluar la prevalencia de estas conductas. En consecuencia, estos esfuerzos se han dirigido en la construcción y diseño de instrumentos para medir la "adicción" (Echeburúa \& Corral, 2010) y el "uso problemático" del internet (Parra et al., 2016; Pulido-Rull, Escoto-de la Rosa \& Gutiérrez-Valdovinos, 2011).

En ese orden de ideas, Pulido-Rull et al. (2011) señalan que este fenómeno forma parte de una serie de conductas problemáticas que, para ser consideradas como tales, deben seguir algunos criterios en el que las personas pueden incurrir en la sustitución de las actividades llevadas a cabo en la vida cotidiana por el uso del internet. Adicionalmente, se presenta una pérdida de control en la dosificación de tiempo de conexión y se reconoce este hecho, surgiendo la anticipación por pensar en la próxima vez que se tenga acceso al internet, además de que recurren a este medio como una forma de escape del mundo real. Así, el uso problemático del internet (UPI) se refiere a "la incapacidad de controlar el tiempo de conexión, relegando e interfiriendo con obligaciones y tareas personales, laborales, familiares, sociales o académicas" (Sánchez-Rodríguez, Ruíz-Palmero y Sánchez-Rivas, 2015, p. 161). En consecuencia, esto puede derivar en problemas de salud física (migrañas, dolor lumbar, sobrepeso u obesidad o descanso insuficiente) o causar malestar de tipo psicológico (Fernández-Villa et al., 2015; Parra et al., 2016).

Adicionalmente, otro riesgo al que se exponen adolescentes y jóvenes durante el uso de las TICs es la violencia que puede surgir entre sus propios grupos de edad (Arnaiz, Cerezo, Giménez \& Maquilón, 2016), conductas que son conocidas como cyberbullying y que se caracterizan por el uso de las TICs para infringir daño de una forma repetitiva e intencionada a una persona que no puede defenderse fácilmente (Crosslin \& Crosslin, 2014; Kokkinos, Antoniadou \& Markos, 2014). En este contexto virtual, los usos de estas herramientas otorgan al abusador cierto anonimato lo que, aunado al menor control parental, supervisión y falta de normas sociales, provocan daños que pueden generar un malestar psicológico mayor (Mateus, Veiga, Costa \& Dores, 2015). Esto, sin olvidar el incremento de una cuantiosa audiencia que en mayoría de las veces, no interviene al presenciar estas situaciones actuando como testigos (Wozencroft, Campbell, Orel, Kimpton \& Leong, 2015) o que la víctima posteriormente invierte su papel por el de un agresor (a) justificando sus acciones con el daño anteriormente sufrido (Crossling \& Crossling, 2014) en vez de hacer uso de estrategias de afrontamiento o solicitar apoyo institucional (Orel, Campbell, Wozencroft, Leong \& Kimpton, 2015).

Asimismo, de acuerdo con Henao-Rodríguez, Martínez y Velasco-Benítez (2015), este fenómeno se puede categorizar por el tipo de maltrato que sufren sus víctimas. Así, por ejemplo: (1) el Flaming, suele producirse en foros públicos (Gahagan, Vaterlaus \& Frost, 2016); (2) el acoso online (Online Harassment) que tiene el propósito de molestar o herir a alguien por medio de mensajes ofensivos y persistentes (Crosslin \& Crosslin, 2014); (3) el robo de identidad (Identity Theft) para hacerse pasar por el usuario (a) y enviar información perjudicial a sus contactos (Prieto, Carrillo \& Lucio, 2015); mientras que (4) la exposición (Outing) se realiza por medio del envío de información privada que no estaba destinada a ser publicada (Ozgur, 2015); (5) la exclusión (Ostracism) a través del bloqueo o eliminación de una lista de amigos (as) o cuando los propios contactos no contestan a sus mensajes y correos electrónicos (López, 2017); (6) la mala información (Missinformation) que surge con la difusión falsa de información de una persona a través de las páginas web, correos, mensajes de texto o mensajería instantánea (López, 2017); (7) el ciber-acoso (Cyberstalking) que implica amenazas repetitivas, acecho e intimidación (Savage \& Tokunaga, 2017) y (8) la golpiza feliz (Happy slapping) cuando abusadores (as) intencionalmente intimidan, golpean o se burlan de sus víctimas mientras son grabadas para publicarse en internet.

Dicho lo anterior es preciso hacer énfasis en que gran parte de los estudios realizados al respecto se enfocan en poblaciones de adolescentes (Gahagan et al., 2016; Mateus et al., 2015), mientras que las investigaciones con poblaciones jóvenes son aún limitados (Henao-Rodríguez et al., 2015). Situación a la que se añade una escasez en el di- 
seño de programas de intervención y prevención sobre el fenómeno (Cunningham et al., 2015; Garaigordobil, 2014; Lawler \& Molluzzo, 2015).

Algo semejante ocurre con el cambio en los tipos de violencia de pareja que experimenta la juventud en relación con el uso inadecuado de las TICs (Durán \& Martínez-Pecino, 2015), por lo que algunos estudios han planteado que el creciente uso de las TICs en la comunicación de las parejas jóvenes ha generado de manera inadecuada, una forma para generar daño a un miembro de la pareja durante sus conflictos o discusiones (Burke \& Martsolf, 2010), un fenómeno que hasta el momento ha sido estudiado insuficientemente a nivel empírico (Borrajo \& Gámez-Guadix, 2015). Es así como la Ciber-violencia de Pareja (CvP) o también conocida como Cyber Dating Abuse, involucra específicamente el uso de las TICs para agredir directamente por medio de amenazas, insultos, humillaciones o denigrar a un miembro de la pareja. Además de otras conductas enfocadas a controlar/monitorear a la pareja actual o acosar a la expareja, después de la ruptura de la relación; tales como las llamadas y mensajes constantes, y el bloqueo o eliminación de la lista de contactos en las redes sociales (Borrajo, Gámez-Guadix \& Calvete, 2015a; Schnurr, Mahatmya \& Basche, 2013; Zweig, Lachman, Yahner \& Dank, 2013).

Estas conductas de maltrato psicológicode acuerdo con Martín, Pazos, Montilla \& Romero (2016), anteceden a las primeras señales de alarma frente a una relación que se irá violentando de manera gradual en las que ambos miembros pueden incurrir de forma recíproca en conductas violentas de este tipo (Borrajo, Gámez-Guadix \& Calvete, 2015b; Zweig, Dank, Yahner \& Lachman, 2013). Al igual que en el caso del cyberbullying, este fenómeno se encuentra más estudiado en adolescentes (Jaen-Cortés, Rivera-Aragón, Reidl-Martínez \& García-Méndez, 2017; Van Ouytsel, Ponnet, Walrave \& Temple, 2016), comparadas con las pocas investigaciones que afirman que la CvP también se encuentra presente en jóvenes universitarios (Strawhun, Adams \& Huss, 2013).

Por todo lo anterior, este estudio cuantitativo se ha desarrollado con un diseño no experimental de corte transversal, con un alcance exploratorio, descriptivo y correlacional, ha sido propuesto para lograr los siguientes tres objetivos: (1) identificar las principales conductas referentes al Uso Problemático del Internet, el Cyberbullying y la Ciber-violencia de Pareja, (2) analizar diferencias por sexo en estas conductas y, por último, (3) explorar la relación entre las variables mencionadas.

\section{Método}

\section{Participantes}

La muestra estuvo constituida por 456 jóvenes, $21,5 \%$ de hombres y $78,5 \%$ de mujeres (procedentes de dos universidades públicas mexicanas. 244 estudiantes de una universidad del estado de Tamaulipas y el resto del alumnado de una Universidad de Puebla $(n=212)$. Las edades estuvieron comprendidas entre los 17 y 30 años $(M=19,86$; $\mathrm{DT}=2,12$ ).

\section{Instrumentos}

Para efectos del estudio se utilizaron tres instrumentos de medición, uno para cada variable de estudio: (a) Uso problemático del internet, (b) cyberbullying y (c) Ciber-violencia de pareja. A pesar de que los instrumentos utilizados cuentan con análisis psicométricos previos, en sus versiones impresas, al utilizarse en una versión on-line se ha decidido evaluar su consistencia interna para la muestra de esta investigación.

(a) El Cuestionario de Uso problemático de Internet (CUPI) de Pulido-Rull et al., (2011), mide el uso problemático del internet en universitarios mexicanos y está constituido por 18 preguntas que se encuentran agrupadas en 5 factores y que en el presente estudio han obtenido consistencias internas aceptables entre las subescalas sustitución $(\alpha=, 70)$, pérdida de control $(\alpha=, 71)$, anticipación $(\alpha=, 78)$, reconocimiento de falta de control $(\alpha=, 60)$ y evasión $(a=, 77)$. La escala de respuesta es tipo Likert con las siguientes opciones: 0 (nunca), 1 (rara vez), 2 (a veces), 3 (seguido) y 4 (muy seguido). 
(b) El Cyberbullying. Screening de acoso entre iguales (Garaigordobil, 2013; 2014) se configura en dos secciones que permiten identificar la existencia y gravedad del (1) bullying "cara a cara" (físico, verbal, social y psicológico), a través de 12 ítems; y (2) conductas de cyberbullying, con 45 reactivos. El screening informa de 3 indicadores para el fenómeno de la perpetración, sufrimiento y atestiguamiento de bullying y ciberbullying. No obstante, en esta investigación solo se ha implementado el screening de ciberbullying obteniendo buenos índices de consistencia interna para cibervictimización $a=, 84$; ciberagresión $a=, 65$; ciberobservación $a=90$. La escala de respuesta es de tipo Likert con las siguientes opciones: 0 (nada), 1 (algunas veces), 2 (bastantes veces) y 3 (siempre).

(c) El Cyber Dating Abuse Questionnaire (C. D. A. Q.), desarrollado por Borrajo, Gámez-Guadix, Pereda y Calvete (2015), evalúa la frecuencia de conductas de ciber-violencia de pareja a través de 20 reactivos de doble naturaleza, uno para victimización y otro para la perpetración de la conducta evaluada, mismos que se encuentran agrupados en 4 factores. Se analizó la consistencia interna del instrumento mediante coeficiente alfa de Cronbach, obteniendo puntuaciones de buenas a excelentes en cada una de las subescalas: victimización de control/monitoreo $(a=, 87)$, perpetración de control/monitoreo $(a=, 84)$, victimización de agresiones directas $(a=, 86)$ y perpetración de agresiones directas $(\alpha=, 77)$. La escala Likert incluye 6 opciones de respuesta: 1 (Nunca), 2 (No en el último año, pero si anteriormente), 3 (Rara vez), 4 (A veces), 5 (Con frecuencia) y 6 (Casi siempre).

\section{Procedimiento}

Los participantes fueron contactados de manera incidental, por muestreo no probabilístico y se aplicaron cuestionarios de autoinforme vía online con una duración aproximada de 20 minutos. El criterio de inclusión fue tener o haber tenido pareja con una duración mínima de un mes.Los estudiantes participantes accedieron voluntariamente una vez que se les informó la naturaleza y objetivos de la investigación, así como del manejo de los datos obtenidos de manera confidencial y anónima. Antes de iniciar la encuesta se incluyó el consentimiento informado electrónico a través de un ítem, en cumplimiento de las recomendaciones del código ético de la Sociedad Mexicana de Psicología (2010).

\section{Resultados}

En primer lugar, para cumplir con el objetivo uno se realizaron análisis descriptivos de las conductas de Uso Problemático de Internet (UPI), cyberbullying y Ciber-violencia de Pareja (CvP); tal como se puede ver en la Tabla 1. Las puntuaciones más altas en relación con el UPI, fueron en la subescala pérdida de control $(M=5,55 ; D T=2,56)$, mientras que la más baja fue la de sustitución $(M=3,06 ; D T=2,85)$. En cuanto al cyberbullying, los valores medios más altos se identificaron para la ciberobservación ( $M=5,42 ; D T=5,92)$, seguida por la ciberagresión $(M=2,71$; $D T=3,53)$, y por último la cibervictimización $(M=, 80 ; D T=1,53)$. En lo que respecta a las conductas de CvP, se presentaron mayores puntuaciones en las subescalas de control/monitoreo, siendo mayor la victimización $(M=13,59 ; D T=7,35)$ que la perpetración reportada $(M=12,77 ; D T=5,55)$. 
Tabla 1.

Estadísticos descriptivos de las subescalas de UPI, cyberbullying y CvP $(N=456)$.

\begin{tabular}{clccc}
\hline \multicolumn{7}{c}{} & Mediana & Media & DE \\
\hline Uso problemático del Internet (UPI) & & & \\
\hline 1 & Sustitución & 2 & 3,06 & 2,85 \\
\hline 2 & Pérdida de control & 5 & 5,55 & 2,56 \\
\hline 3 & Anticipación & 4 & 4,73 & 3,18 \\
\hline 4 & Reconocimiento de falta de control & 4 & 4,40 & 2,40 \\
\hline 5 & Evasión & 4 & 4,02 & 2,83 \\
\hline Cyberbullying & & & \\
\hline 6 & Cibervictimización & 2 & 2,71 & 3,53 \\
\hline 7 & Ciberagresión & 0 &, 80 & 1,53 \\
\hline 8 & Ciberobservación & 4 & 5,42 & 5,92 \\
\hline Ciber-violencia de Pareja (CvP) & & & \\
\hline 9 & Victimización de control/monitoreo & 11 & 13,59 & 7,35 \\
\hline 10 & Perpetración de control/monitoreo & 11 & 12,77 & 5,55 \\
\hline 11 & Victimización de agresiones directas & 11 & 12,24 & 3,56 \\
\hline 12 & Perpetración de agresiones directas & 11,82 & 2,31 \\
\hline
\end{tabular}

Fuente: Elaboración propia.

Adicionalmente, con el fin de explorar las conductas realizadas con mayor frecuencia por los jóvenes, se analizaron las respuestas sobresalientes en cada variable del estudio. Así, por parte del UPI resaltaron los reactivos 2 (¿Con qué frecuencia te conectas a internet a pesar de tus esfuerzos por no hacerlo?), 8 (¿Has pensado querer usar menos internet?) y 1 (¿Con qué frecuencia te ha resultado difícil dejar de usar internet?), ver Tabla 2.

Tabla 2.

Distribución de frecuencias por ítem sobre UPI $(\mathrm{N}=456)$.

\begin{tabular}{cccccc}
\hline \multirow{2}{*}{$\begin{array}{c}\text { Número de } \\
\text { pregunta }\end{array}$} & Nunca & Rara vez & A veces & Seguido & Muy seguido \\
\cline { 2 - 6 } & $f(\%)$ & $f(\%)$ & $f(\%)$ & $f(\%)$ & $f(\%)$ \\
\hline 2 & $32(7,01)$ & $108(23,68)$ & $139(30,48)$ & $129(28,29)$ & $48(10,53)$ \\
\hline 8 & $66(14,47)$ & $84(18,42)$ & $188(41,23)$ & $77(16,89)$ & $41(8,99)$ \\
\hline 1 & $36(7,89)$ & $139(30,48)$ & $168(36,84)$ & $83(18,20)$ & $30(6,58)$ \\
\hline
\end{tabular}

Fuente: Elaboración propia. 
Mientras que en los ítems sobre cyberbullying, se halló mayor variabilidad en los reactivos: 34 (¿Has visto difundir fotos o vídeos privados o comprometedores de alguien utilizando el teléfono celular o internet), 26 (¿Has visto fotos o vídeos de alguien que hayan sido modificadas para difundirlas mediante redes sociales o páginas web - por ejemplo, You Tube - y humillaste o te reíste de él?) y 1 (¿Te han enviado mensajes ofensivos e insultantes mediante el teléfono celular?), véase Tabla 3.

Tabla 3.

Distribución de frecuencias por ítem sobre cyberbullying $(N=456)$.

\begin{tabular}{ccccc}
\hline \multirow{2}{*}{$\begin{array}{c}\text { Número } \\
\text { de reactivo }\end{array}$} & Nada & Algunas veces & Bastantes veces & Siempre \\
\cline { 2 - 5 } & $f(\%)$ & $f(\%)$ & $f(\%)$ & $f(\%)$ \\
\hline 34 & $266(58,33)$ & $125(27,41)$ & $58(12,72)$ & $7(1,54)$ \\
\hline 26 & $345(75,66)$ & $79(17,32)$ & $28(6,14)$ & $4(0,88)$ \\
\hline 1 & $278(60,96)$ & $153(33,55)$ & $24(5,26)$ & $1(0,22)$ \\
\hline
\end{tabular}

Fuente: Elaboración propia.

Respecto a la CvP, sobresalieron los ítems: 7a $(\mathrm{Mi}$ pareja o expareja ha controlado la hora de mi última conexión en aplicaciones del móvil), 7b (He controlado la hora de la última conexión de mi pareja o expareja en aplicaciones del móvil), 5a
(Mi pareja o expareja ha utilizado mis contraseñas del teléfono, redes sociales y correo para curiosear mis mensajes y/o contactos sin mi permiso) y $11 \mathrm{~b}$ (He revisado las redes sociales, WhatsApp o correo de mi pareja sin su permiso), ver Tabla 4.

Tabla 4.

Distribución de frecuencias por ítem sobre CVP $(\mathrm{N}=456)$.

\begin{tabular}{ccccccc}
\hline \multirow{2}{*}{$\begin{array}{c}\text { Número } \\
\text { de ítem }\end{array}$} & Nunca & $\begin{array}{c}\text { No en el último } \\
\text { año, pero si } \\
\text { anteriormente }\end{array}$ & Rara vez & A veces & $\begin{array}{c}\text { Con } \\
\text { frecuencia }\end{array}$ & Casi siempre \\
\cline { 2 - 7 } & $f(\%)$ & $f(\%)$ & $f(\%)$ & $f(\%)$ & $f(\%)$ & $f(\%)$ \\
\hline $7 \mathrm{a}$ & $342(75)$ & $35(7,68)$ & $38(8,33)$ & $19(4,17)$ & $7(1,54)$ & $15(3,29)$ \\
\hline $5 \mathrm{a}$ & $332(72,81)$ & $37(8,11)$ & $38(8,33)$ & $29(6,36)$ & $10(2,19)$ & $10(2,19)$ \\
\hline $7 \mathrm{~b}$ & $341(74,78)$ & $35(7,68)$ & $41(8,99)$ & $22(4,82)$ & $7(1,54)$ & $10(2,19)$ \\
\hline $11 \mathrm{~b}$ & $320(70,18)$ & $56(12,28)$ & $59(12,94)$ & $11(2,41)$ & $3(0,66)$ & $7(1,54)$ \\
\hline
\end{tabular}

Fuente: Elaboración propia. 
Con respecto al objetivo dos, se utilizó la prueba $\mathrm{U}$ de Mann-Whitney para determinar las diferencias por sexo en las subescalas que componen las variables estudiadas, donde solo se encontraron diferencias estadísticamente significativas en la sustitución, ciberagresión y en la victimización de agresiones directas, tal como se muestra en la Tabla 5.

Tabla 5.

Descriptivos y diferencias por sexo en las subescalas de UPI, cyberbullying y CvP ( $N=456)$.

\begin{tabular}{|c|c|c|c|c|c|c|c|c|c|c|}
\hline & \multicolumn{4}{|c|}{ Hombres $(n=98)$} & \multicolumn{4}{|c|}{ Mujeres $(n=358)$} & \multirow[b]{2}{*}{$u$} & \multirow[b]{2}{*}{ valor $\mathrm{p}$} \\
\hline & Md & $M$ & $D T$ & $\begin{array}{l}\text { Rango } \\
\text { promedio }\end{array}$ & Md & $M$ & $D T$ & $\begin{array}{c}\text { Rango } \\
\text { promedio }\end{array}$ & & \\
\hline \multicolumn{11}{|c|}{ Uso problemático del Internet (UPI) } \\
\hline 1 & 3 & 3,49 & 2,87 & 253,09 & 2 & 2,94 & 2,84 & 221,77 & 15132 & ,035* \\
\hline 2 & 5 & 5,57 & 2,78 & 229,1 & 6 & 5,54 & 2,51 & 228,34 & 17483,5 & 0,959 \\
\hline 3 & 4 & 4,66 & 3,10 & 225,56 & 5 & 4,75 & 3,21 & 229,3 & 17254 & 0,802 \\
\hline 4 & 4 & 4,24 & 2,27 & 221,42 & 4 & 4,45 & 2,44 & 230,44 & 16848 & 0,545 \\
\hline 5 & 3 & 3,76 & 2,65 & 217,78 & 4 & 4,10 & 2,88 & 231,43 & 16491,5 & 0,361 \\
\hline \multicolumn{11}{|c|}{ Cyberbullying } \\
\hline 6 & 2 & 3,04 & 3,39 & 245,55 & 2 & 2,62 & 3,57 & 223,83 & 15871,5 & 0,14 \\
\hline 7 & 1 & 1,43 & 2,41 & 269,34 & 0 & 0,62 & 1,13 & 217,32 & 13540 &, $000^{* *}$ \\
\hline 8 & 5 & 6,48 & 6,67 & 248,09 & 4 & 5,13 & 5,68 & 223,14 & 15622,5 & 0,094 \\
\hline \multicolumn{11}{|c|}{ Ciber-violencia de Pareja (CvP) } \\
\hline 9 & 11 & 14,23 & 8,25 & 237,95 & 10 & 13,41 & 7,02 & 225,91 & 16615,5 & 0,402 \\
\hline 10 & 11 & 12,7 & 5,93 & 224,51 & 11 & 12,79 & 5,45 & 229,59 & 17151 & 0,727 \\
\hline 11 & 11 & 13,28 & 6,19 & 253,25 & 11 & 11,96 & 2,31 & 221,72 & 15116,5 &, $010^{* *}$ \\
\hline 12 & 11 & 12,17 & 3,94 & 232,16 & 11 & 11,72 & 1,60 & 227,5 & 17183 & 0,696 \\
\hline
\end{tabular}

1) Sustitución, 2) Pérdida de control, 3) Anticipación, 4) Reconocimiento de falta de control, 5) Evasión, 6) Cibervictimización, 7) Ciberagresión, 8) Ciberobservación, 9) Victimización de control/monitoreo, 10) Perpetración de control/monitoreo, 11) Victimización de agresiones directas, 12) Perpetración de agresiones directas.

Fuente: elaboración propia. 
Por último, para alcanzar el tercer objetivo y determinar la asociación entre las variables estudiadas se realizó el análisis de correlación de Spearman en la muestra total, encontrando bajas pero significativas asociaciones positivas en algunas variables (véase Tabla 6).

Correlaciones entre el UPI con Cyberbullying: en las subescalas de sustitución con la cibervictimización (rho $=, 233 ; \mathrm{p}<, 01$ ), la evasión con la ciberobservación (rho $=, 209 ; \mathrm{p}<, 01$ ) y la sustitución con la ciberagresión (rho $=, 205 ; p<, 01$ ).

Asociaciones entre el UPI y la CVP: en la evasión con la perpetración de agresiones directas (rho $=, 244 ; \mathrm{p}<, 01)$, la pérdida de control con la perpetración de control/monitoreo ( $r$ ho = ,200; $\mathrm{p}<, 01)$, la sustitución con la victimización de agresiones directas (rho $=, 194 ; p<, 01)$ y en la evasión con la victimización del control/monitoreo $($ rho $=, 182 ; \mathrm{p}<, 01)$.

Relaciones entre el cyberbullying y la CVP: en la cibervictimización con la perpetración de control/monitoreo (rho = ,282; $\mathrm{p}<, 01$ ), la ciberagresión con la victimización de agresiones directas (rho $=, 245 ; p<, 01)$ y en la ciberobservación con la victimización de control/monitoreo (rho = ,232; $\mathrm{p}<, 01)$.

Tabla 6.

Correlaciones entre UPI, Cyberbullying y CvP $(\mathrm{N}=456)$.

\begin{tabular}{|c|c|c|c|c|c|c|c|c|c|c|c|c|}
\hline & \multicolumn{5}{|c|}{ UPI } & \multicolumn{3}{|c|}{ Cyberbullying } & \multicolumn{4}{|c|}{ CvP } \\
\hline & 1 & 2 & 3 & 4 & 5 & 6 & 7 & 8 & 9 & 10 & 11 & 12 \\
\hline 1 & 1 & & & & & & & & & & & \\
\hline 2 &, $528^{* *}$ & 1 & & & & & & & & & & \\
\hline 3 & ,648** &, $703^{* *}$ & 1 & & & & & & & & & \\
\hline 4 &, $491^{* *}$ &, $635^{* *}$ &, $663^{* *}$ & 1 & & & & & & & & \\
\hline 5 &, $597^{* *}$ &, $568^{* *}$ & ,638** &, $534^{* *}$ & 1 & & & & & & & \\
\hline 6 &, $233^{* *}$ &, $162^{* *}$ &, $183^{* *}$ &, $198^{* *}$ &, $193^{* *}$ & 1 & & & & & & \\
\hline 7 &, $205^{* *}$ & ,111* &, $122^{* *}$ & ,079 &, $177^{* *}$ &, $384^{* *}$ & 1 & & & & & \\
\hline 8 &, $146^{* *}$ &, $117^{*}$ &, $157^{* *}$ &, $100^{*}$ & ,209** &, $361^{* *}$ &, $365^{* *}$ & 1 & & & & \\
\hline 9 &, $143^{* *}$ &, $170^{* *}$ &, $135^{* *}$ &, $133^{* *}$ &, $182^{* *}$ &, $277^{* *}$ &, $151^{* *}$ &, $232^{* *}$ & 1 & & & \\
\hline 10 &, $160^{* *}$ &, $200^{* *}$ &, $173^{* *}$ &, $143^{* *}$ &, $183^{* *}$ &, $282^{* *}$ &, $168^{* *}$ & ,190** & ,690** & 1 & & \\
\hline 11 &, $194^{* *}$ &, $174^{* *}$ &, $124^{* *}$ &, $141^{* *}$ &, $186^{* *}$ &, $272^{* *}$ &, $245^{* *}$ &, $219^{* *}$ &, $473^{* *}$ &, $336^{* *}$ & 1 & \\
\hline 12 &, $203^{* *}$ &, $154^{* *}$ &, $186^{* *}$ &, $127^{* *}$ &, $244^{* *}$ &, $195^{* *}$ &, $223^{* *}$ &, $153^{* *}$ &, $351^{* *}$ &, $356^{* *}$ &, $584^{* *}$ & 1 \\
\hline \multicolumn{13}{|c|}{ Nota. ${ }^{*} \mathrm{p}<, 05 \mathrm{y}{ }^{* *} \mathrm{p}<, 01$} \\
\hline & $\begin{array}{l}\text { tución, } \\
\text { zación, } \\
\text { /monit }\end{array}$ & $\begin{array}{l}\text { 2) Pérdi } \\
\text { 7) Ciber } \\
\text { reo, 11) }\end{array}$ & $\begin{array}{l}\text { de cor } \\
\text { esión, } \\
\text { timiza }\end{array}$ & $\begin{array}{l}\text { ol, 3) } \\
\text { Ciberc } \\
\text { on de }\end{array}$ & $\begin{array}{l}\text { icipaci } \\
\text { ervaci }\end{array}$ & $\begin{array}{l}\text { 4) } \mathrm{Re} \\
\text { 9) } \mathrm{Vic}\end{array}$ & $\begin{array}{l}\text { locimie } \\
\text { nizaciór } \\
\text { 2) Perp }\end{array}$ & $\begin{array}{l}\text { o de fa } \\
\text { de cont }\end{array}$ & $\begin{array}{l}\text { de cont } \\
\text { monito }\end{array}$ & $\begin{array}{l}\text { ol, 5) } E \\
o, 10) \\
\text { s direc }\end{array}$ & $\begin{array}{l}\text { sión, } 6 \\
\text { rpetra }\end{array}$ & $\begin{array}{l}\text { ber- } \\
\text { n de }\end{array}$ \\
\hline
\end{tabular}

Fuente: Elaboración propia. 


\section{Discusión}

Con respecto al primer objetivo de esta investigación (identificar las principales conductas referentes al UPI, cyberbullying y (vP), es preciso señalar que los resultados obtenidos sobre el Uso problemático del Internet (UPI) un 38,82 \% de los y las jóvenes participantes en este estudio indicaron usar el internet con una frecuencia de "seguido" y "muy seguido", a pesar de sus intentos por no hacerlo. En el estudio realizado por García et al., (2008) con estudiantes universitarios plantearon que los jóvenes con mayor frecuencia de uso del internet ( 5 a 7 veces por semana) se diferenciaron significativamente del resto presentando medias más elevadas en todas las subescalas de uso y abuso de internet. También, se presentó un incremento en el uso del teléfono móvil y el ordenador con acceso a internet, como apunta la investigación de Arnaiz, Cerezo, Giménez y Maquilón (2016), quienes obtuvieron una frecuencia regular de uso del internet de 1 a 2 horas por día; o el trabajo de Molero et al., (2014), quienes enfatizan que sus participantes acceden a internet entre 2 y 4 veces en un mismo día. Por tanto, podría esperarse que a mayor frecuencia de uso de internet habrá mayor probabilidad de presentar conductas de UPI.

Por otra parte, como en cualquier conducta problemática, es necesario reconocer cuando ésta se presenta a fin de modificarla, algo que no siempre se logra. En ese sentido, en la presente investigación un $25,88 \%$ de los y las participantes no parecen identificar posibles riesgos o problemas graves asociados a la utilización del internet de manera excesiva y quizás por esto no han considerado que con dificultad podrían dejar de usar esta herramienta. Espinar y López (2009) sugieren al respecto que los jóvenes creen que conocen el manejo de estas herramientas, se sienten seguros en el uso que le dan y no parecen preocuparse de desarrollar una dependencia a las mismas. En esa línea, Rial, Gómez, Braña y Varela (2014), en un estudio con población adolescente, encontraron que los participantes reconocían que la "gente de su edad" hace un uso poco recomendable de la red (exoactitud negativa), no obstante, cuando se trataba de realizar atribuciones acerca de sí mismos, informaban de un posible riesgo de dependencia muy bajo y de un nivel de apego hacia Internet y redes sociales moderado (endoactitud positiva).

Por otra parte, el reconocimiento de la adicción - conductas problemáticas es un primer paso para la moderación de estas. Así, los resultados de nuestro estudio parecen apoyar el hecho de que las conductas de uso inadecuado del internet pueden ser identificadas por los jóvenes. No obstante, a pesar de sus esfuerzos parece que les resulta difícil dejar de hacerlo; lo que se puede corroborar en el 36,84 \% (a veces), 18,20 \% (bastantes veces) y un 6,58 \% (siempre) de la muestra que ha llegado a considerar tener dificultad para dejar de usar el internet, comparado con el $30,48 \%$ que lo ha pensado rara vez o el $7,89 \%$ que nunca ha tomado en cuenta esta situación.

Por otro lado, con referencia a los hallazgos en el cyberbullying, en el presente estudio se encontró una mayor frecuencia de ocurrencia en algunas conductas de testificación, seguidas por la perpetración y en menor cantidad, se encuentra presente la victimización. Algo similar a lo encontrado en el estudio de Gahagan et al., (2016) realizado con universitarios, en donde se encontró una prevalencia de $61 \%$ de testigos, $46 \%$ de agresores y un $19 \%$ de víctimas; y que contrastan con las investigaciones de Mateus et al., (2015) y Prieto et al., (2015), quienes reportaron una alta prevalencia de cibervíctimas respecto a las puntuaciones de perpetración; no obstante, en aquellos estudios no se evaluó la testificación.

En último término, con relación a las conductas de CVP se encontró un porcentaje inferior al $30 \%$ de conductas asociadas a la victimización y perpetración del control/monitoreo y agresiones directas por medio del teléfono móvil e internet hacia la pareja o expareja; datos que son consistentes con los resultados del estudio exploratorio y cualitativo de Borrajo y Gámez-Guadix (2015), en el que concluyen que las TICs son utilizadas por las parejas o exparejas para llevar a cabo conductas de control y amenazas. En la misma dirección Borrajo, Gámez-Guadix y Calvete (2015), en una investigación posterior, hallaron que un 
$50 \%$ de sus participantes han sido víctimas de algunos tipos de CvP en los últimos 6 meses y el tipo de violencia más común fueron comportamientos para controlar a la pareja. Algo de suma importancia si se considera que al parecer este medio se ha transformado en una herramienta de control durante una relación de pareja donde sus participantes reconocen este hecho como "normal y esperable", asumiendo que esta dinámica forma parte de la convivencia íntima con la otra persona (Martín et al., 2016).

En cuanto al segundo objetivo del estudio (analizar las diferencias por sexo en esos tipos de conductas), solamente se encontró que los hombres presentaron mayor tendencia que las mujeres a optar por sustituir alguna actividad cotidiana por el uso del internet, siendo solo un indicador de varios, que miden el UPI. Estos resultados concuerdan con los hallazgos de Puerta-Cortés y Carbonell (2014) y Ruíz-Olivares et al. (2010), en donde los varones presentaron mayor porcentaje de conductas de UPI; pero también contrapuestos con investigaciones como la de Fernández-Villa et al. (2015) quienes no encontraron diferencias significativas en función del sexo.

En lo concerniente al cyberbulling, solamente se encontraron diferencias significativas en la subescala de ciberagresión; resultados que coinciden parcialmente con los de Kokkinos et al. (2014) y de Ozgur (2015), que sugieren mayores puntuaciones de ciberagresión y cibervictimización en los hombres, que en las mujeres. No obstante, estos datos difieren de los presentados por Wozencroft et al. (2015) y López (2017), quienes no encontraron diferencias estadísticamente significativas por sexo.

Algo similar se presentó en el caso de la CvP, en donde sólo se hallaron diferencias estadísticamente significativas en la victimización de agresiones directas (CVP) reportadas en mayor cantidad por los varones. Las diferencias por sexo parecen cuestionables, esto se puede observar en los resultados distintos presentados al respecto en otros estudios que planearon una mayor prevalencia de victimización en varones, tanto en poblaciones de adolescentes, como de universitarios (Jaen-Cortés et al., 2017; Strawhun et al., 2013); y aquellos que plantean la victimización mayor entre las chicas (Schnurr et al., 2013; Zweig, Lachman, Yahner \& Danck, 2013).

Pese a estos hallazgos, de manera global es preciso enfatizar que, al encontrarse solo una diferencia estadísticamente significativa en una subescala de cada variable de estudio, la interpretación de estos datos sebe ser cautelosa y, mejor aún, profundizarse en futuros estudios. Estas inconsistencias reportadas en la presente investigación podrían originarse, entre otras cosas, por utilización de autoinformes como instrumentos de medición, por lo que estos hallazgos contrastan con otros estudios que han planteado objetivos similares (Fernández-Villa et al. 2015; Jaen-Cortés et al., 2017; López, 2017; Strawhun et al., 2013; Wozencroft et al. 2015), sin olvidar la posibilidad de que los mismos han empleado una metodología distinta y la asociación con otras variables.

Por último, en lo concerniente al tercer objetivo (explorar la relación entre las variables antes mencionadas) de esta investigación, inicialmente se hallaron bajas pero significativas correlaciones entre algunas conductas que caracterizan al UPI con la mayor parte de subescalas del cyberbullying; estos datos pueden presentar un antecedente que contribuye a la necesidad de investigar estas relaciones con población universitaria, para conocer a profundidad los niveles de relación entre las variables antes mencionadas.

Asimismo, en el presente estudio se encontró una baja pero significativa relación entre comportamientos del UPI y conductas asociadas a la CvP, siendo hallazgos que aún requieren de mayor profundización en investigaciones posteriores.

Además, los datos obtenidos en sugieren una posible asociación entre conductas de cyberbullying con la CvP, lo que representa un aporte empírico respecto a la frecuencia de ocurrencia de ambos fenómenos. Al respecto es preciso recordar los estudios de Crosslin y Crosslin (2014), quienes hallaron la presencia de cibervictimización originada de la ruptura de una diada; o el de Durán y Martínez-Pecino (2015) en el que identificaron una posible relación entre haber sido victimizado por 
su pareja a través del uso de las TICs y el ejercicio del ciberacoso.

\section{Conclusiones}

Los datos aportados por esta investigación indican que el uso de las TICs con acceso a internet, principalmente, forma parte de la vida cotidiana de los jóvenes. Además, se detectó la presencia de conductas que pueden generar daño, como el uso problemático del internet en relación con interacciones violentas entre pares (cyberbullying) y la ciber-violencia de pareja. Así se pudo observar que las conductas de uso problemático de internet, el cyberbullying y la ciber-violencia de pareja se encuentran correlacionadas en niveles muy bajos. Por lo que estos resultados buscan coadyuvar a un mejor entendimiento de dichos fenómenos y así, favorecer a la propuesta o mejora de programas de prevención de las nuevas tendencias de ciberconducta a través de la promoción de una sana convivencia por el uso adecuado de las TICs.

Sin detrimento de lo anterior, es preciso enfatizar la naturaleza exploratoria de la presente investigación que cuenta con limitaciones propias del diseño de estudio y el sesgo de la deseabilidad social que se encuentra inherente al empleo de autoinformes. Por otra parte, existe dificultad para generalizar los resultados debido a la procedencia de la muestra y su representatividad como unidad de análisis en la población universitaria mexicana. Futuros estudios podrían ampliar y diversificar la muestra incluyendo estudiantes de otras universidades del país.

\section{Referencias}

Arnaiz, P., Cerezo, F., Giménez, A. M. \& Maquilón, J. J. (2016). Conductas de ciberadicción y experiencias de cyberbullying entre adolescentes. Anales de Psicología, 32(3), 761-769. doi:10.6018/analesps.32.3.217461

Borrajo, E. \& Gámez-Guadix, M. (2015). Comportamientos, motivos y reacciones asociadas a la victimización del abuso online en el no- viazgo: un análisis cualitativo. Revista de Victimología, 2, 73-95. doi:10.12827/RVJV.2.04

Borrajo, E., Gámez-Guadix, M. \& Calvete, E. (2015a). Cyber dating Abuse: Prevalence, Context, and Relationship with offline Dating Aggression. Psychological Reports: Relationships \& Communications, 116(2), 565-585. doi:10.2466/21.16.PR0.116k22w4

Borrajo, E., Gámez-Guadix, M. \& Calvete, E. (2015b). Justification beliefs of violence, myths about love and cyber dating abuse. Psicothema, 27(4), 327-333. doi:10.7334/psicothema2015.59

Borrajo, E., Gámez-Guadix, M., Pereda, N. \& Calvete, E. (2015). The development and validation of the cyber dating abuse questionnaire among young couples. Computers in Human Behavior, 48, 358-365. doi:10.1016/j. chb.2015.01.063

Burke, C. \& Martsolf, D. S. (2010). The role of electronic communication technology in adolescent dating violence. Journal of Child and Adolescent Psychiatric Nursing. 23(3), 133142. doi:10.1111/j.1744-6171.2010.00235.x

Carruth, K. A. \& Ginsburg, H. J. (2014). Social networking and privacy attitudes among college students. Psychology, Society, \& Education, 6(2), 82-93. doi:10.25115/psye.v6i2.510

Castaño-Muñoz, J. \& Senges, M. (2011). Youth-culture or student-culture? The internet use intensity divides among university students and the consequences for academic performance. Estudios sobre Educación, 20, 203-231.

Crosslin, K. \& Crosslin, M. (2014). Cyberbullying at a Texas University - A Mixed Methods Approach to Examining Online Aggression. Texas Public Health Journal, 66(3), 26-31.

Cunningham, C. E., Chen, Y., Vaillancourt, T., Rimas, H., Deal, K., Cunningham, L. J. \& Ratcliffe, J. (2015). Modeling the anti-cyberbullying preferences of university students: adaptive 
choice-based conjoint analysis. Aggressive Behavior, 41, 369-385. doi:10.1002/ab.21560

Durán, M. \& Martínez-Pecino, R. (2015). Ciberacoso mediante teléfono móvil e Internet en las relaciones de noviazgo entre jóvenes. Comunicar, 22(44), 159-167. doi:10.3916/C442015-17

Echeburúa, E. \& De Corral, P. (2010). Adicción a las nuevas tecnologías y las redes sociales: un nuevo reto. Adicciones, 22(2), 91-96. doi:10.20882/adicciones.196

Espinar, E. \& López, C. (2009). Jóvenes y adolescentes ante las nuevas tecnologías: percepción de riesgos. Athenea Digital, 16, 1-20. doi:10.5565/rev/athenead/v0n16.509

Fernández-Villa, T., Alguacil, J., Almaraz, A., Cancela, J. M., Delgado-Rodríguez, M., GarcíaMartín, M., Jiménez-Mejías, E., Llorca, J., Molina, A. J., Ortíz, R., Valero-Juan, L. F. \& Martín, V. (2015). Problematic Internet Use in University Students: associated factors and differences of gender. Adicciones, 27(4), 265275. doi:10.20882/adicciones.751

Gahagan, K., Vaterlaus, J. M. \& Frost, L. R. (2016). College student cyberbullying on social networking sites: Conceptualization, prevalence, and perceived bystander responsibility. Computers Human Behavior, 55, 1097-1105. doi:10.1016/j.chb.2015.11.019

Garaigordobil, M. (2013). Cyberbullying. Screening del acoso entre iguales. Screening del acoso escolar presencial (bullying) y tecnológico (cyberbullying). Madrid: TEA.

Garaigordobil, M. (2014). Cyberbullying. Screening de acoso entre iguales: descripción y datos psicométricos. International Journal of Developmental and Educational Psychology, 4(1), 311-318. doi:10.17060/ijodaep.2014.n1.v4.617

García, J. A., Terol, M. C., Nieto, M., Lledó, A., Sánchez, S., Martín-Aragón, M. \& Sitges, E. (2008). Uso y abuso de Internet en jóvenes universitarios. Adicciones, 20(2), 131-142. doi:10.20882/adicciones. 277

Henao-Rodríguez, D. P., Martínez, C. \& VelascoBenítez, C. A. (2015). Bullying Universitario. Revista Gastrohnup, 17(1), 58-62.

Herrera-Batista, M. A. (2009). Disponibilidad, uso y apropiación de las tecnologías por estudiantes universitarios en México: Perspectivas para una incorporación innovadora. Revista Iberoamericana de Educación, 6(48), 1-9.

Jaen-Cortés, C. I., Rivera-Aragón, S., Reidl-Martínez, L. M. \& García-Méndez, M. (2017). Violencia de pareja a través de medios electrónicos en adolescentes mexicanos. Acta de Investigación Psicológica, 7, 2593-2605. doi:10.1016/j.aipprr.2017.01.001

Kokkinos, C. M., Antoniadou, N. \& Markos, A. (2014). Cyber-bullying: An investigation of the psychological profile of university student participants. Journal of Applied Developmental Psychology, 35, 204-214. doi:10.1016/j. appdev.2014.04.001

Lawler, J. P. \& Molluzzo, J. C. (2015). A comprehensive survey on student perceptions of cyberbullying at a Mayor Metropolitan University. Contemporary Issues in Education Research, 8(3), 159-170. doi:10.19030/cier. v8i3.9347

López, M. G. (2017). Acoso escolar y cibernético en estudiantes universitarios. Revista de Investigación en Educación, 1(15), 11-26.

Martín, A., Pazos, M., Montilla, M. V. C. \& Romero, C. (2016). Una modalidad actual de violencia de género en parejas de jóvenes: las redes sociales. Educación XXI, 19(2), 405-429. doi:10.5944/educXX1.13934

Mateus, S., Veiga, A. M., Costa, P. \& Dores, M. J. (2015). Cyberbullying: The hidden side of college students. Computers in Human Behavior, 43, 167-182. doi:10.1016/j.chb.2014.10.045 
Molero, M. M., Martos, A., Cardila, F., Barragán, A. B., Pérez-Fuentes, M. C., Gázquez, J. J. \& Rosales-Nieto, J. G. (2014). Uso de internet y redes sociales en estudiantes universitarios. European Journal of Child Development, Education and Psychopathology, 2(3), 81-96. doi:10.1989/ejpad.v2i3.24

Odaci, H. \& Kalkan, M. (2010). Problematic Internet use, loneliness and dating anxiety among young adult university students. Computers \& Education, 55, 1091-1097. doi:10.1016/j.compedu.2010.05.006

Orel, A., Campbell, M., Wozencroft, K., Leong, E. \& Kimpton, M. (2015). Exploring University Students' Coping Strategy Intentions for Cyberbullying. Journal of Interpersonal Violence, 32(3), 1-17. doi:10.1177/0886260515586363

Ozgur, H. (2015). Exploring the distance education students' cyberbullying, cybervictimization and cyberbullying sensibility levels. Turkish Online Journal of Distance Education (TOJ$D E), 16(4), 3-17$. doi:10.17718/tojde. 07460

Parra, V., Vargas, J. I., Zamorano, B., Peña, F., Velázquez, Y., Ruiz, L. \& Monreal, O. (2016). Adicción y factores determinantes en el uso Problemático del internet, en una muestra de Jóvenes universitarios EDUTEC. Revista Electrónica de Tecnología Educativa, 56, 60-73. doi:10.21556/edutec.2016.56.741

Prieto, M. T., Carrillo, J. C. \& Lucio, L. A. (2015). Violencia virtual y acoso escolar entre estudiantes universitarios: el lado oscuro de las redes sociales. Innovación Educativa, 15(68), 33-47.

Puerta-Cortés, D. X. \& Carbonell, X. (2013). Uso problemático de Internet en una muestra de estudiantes universitarios colombianos. Avances en Psicología Latinoamericana, 31(3), 620-631.

Puerta-Cortés, D. X. \& Carbonell, X. (2014). El modelo de los cinco grandes factores de personalidad y el uso problemático de Internet en jóvenes colombianos. Adicciones, 26(1), 54-61. doi:10.20882/adicciones.131

Pulido-Rull, M. A., Escoto-de la Rosa, R. \&Gutiérrez-Valdovinos, D. M. (2011). Validez y Confiabilidad del Cuestionario de Uso Problemático de Internet (CUPI). Journal of Behavior, Health \& Social Issues, 3(1), 25-34. doi:10.5460/jbhsi.v3.1.27681

Rial, A., Gómez, P., Braña, T. \& Varela, J. (2014). Actitudes, percepciones y uso de Internet y las redes sociales entre los adolescentes de la comunidad gallega (España). Anales de Psicología, 30(2), 642-655. doi:10.6018/analesps.30.2.159111

Ruíz-Olivares, R., Lucena, V., Pino, M. J. \& Herruzo, J. (2010). Análisis de comportamientos relacionados con el uso/abuso de internet, teléfono móvil, compras y juego en estudiantes universitarios. Adicciones, 22(4), 301-310. doi:10.20882/adicciones.171

Sánchez-Rodríguez, J., Ruíz-Palmero, J. \& Sánchez-Rivas, E. (2015). Uso problemático de las redes sociales en estudiantes universitarios. Revista Complutense de Educación, 26(Especial: TIC en Educación), 159-174. doi:10.5209/ rev_RCED.2015.v26.46360

Savage, M. W. \& Tokunaga, R. S. (2017). Moving toward a theory: Testing an integrated model of cyberbullying perpetration, aggression, communication skills, and internet self-efficacy. Computers in human Behavior, 71, 353361. doi:10.1016/j.chb.2017.02.016

Schnurr, M., Mahatmya, D. \& Basche, R. A. (2013). The Role Of Dominance, Cyber Aggression Perpetration, And Gender On Emerging Adults' Perpetration Of Intimate Partner Violence. Psychology of Violence, 3(1), 70-83. doi:10.1037/a0030601

Sociedad Mexicana de Psicología (2010). Código ético del Psicólogo. México, D.F.: Trillas.

Strawhun, J., Adams, N. \& Huss, M. T. (2013). The Assessment of Cyberstalking: An Expanded 
Examination Including Social Networking, Attachment, Jealousy, and Anger in Relation to Violence and Abuse. Violence and Victims, 28(4), 715-730. doi:10.1891/0886-6708.1100145

Vázquez, L. (2016). Las redes sociales online en España 2012-2016: mucho más que una herramienta comunicativa. Revista de Comunicación de la SEECl, 41(20), 1-16. doi:10.15.198/ seeci.2016.41.01-16

Van Ouytsel, J., Ponnet, K., Walrave, M. \& Temple, J. R. (2016). Adolescent cyber dating abuse victimization and its associations with substance use, and sexual behaviors. Public Health, 135, 147-151. doi:10.1016/j. puhe.2016.02.011
Wozencroft, K., Campbell, M., Orel, A., Kimpton, M. \& Leong, E. (2015). University students' intentions to report cyberbullying. Australian Journal of Educational \& Developmental Psychology, 15, 1-12.

Zweig, J. M., Dank, M., Yahner, J. \& Lachman, P. (2013). The rate of cyber dating abuse among teens and how it relates to other forms of teen dating violence. Journal of Youth and Adolescence, 42, 1063-1077. doi:10.1007/ s10964-013-9922-8

Zweig, J. M., Lachman, P., Yahner, J. \& Dank, M. (2013). Correlates of Cyber Dating Abuse Among Teens. Journal of Youth and Adolescence, 43(8), 1306-1321. doi:10.1007/s10964013-0047-x 